\title{
Reversibility of Airflow Obstruction by Hypoglossus Nerve Stimulation in Anesthetized Rabbits
}

\author{
François Bellemare, Matteo Pecchiari, Monica Bandini, Mohamad Sawan, and Edgardo D'Angelo
}

Laboratoire du sommeil, Service de pneumologie, Centre Hospitalier de l'Université de Montréal (CHUM), Hôtel-Dieu, and Laboratoire PolyStim, École Polytechnique, Montréal, Quebec, Canada; and Istituto di Fisiologia Umana I, Università degli Studi di Milano, Milan, Italy

\begin{abstract}
Rationale: Anesthesia-induced uncoupling of upper airway dilating and inspiratory pump muscles activation may cause inspiratory flow limitation, thereby mimicking obstructive sleep apnea/hypopnea. Objectives: Determine whether inspiratory flow limitation occurs in spontaneously breathing anesthetized rabbits and whether this can be reversed by direct hypoglossal nerve stimulation and by the application of continuous positive airway pressure. Methods: Ten New Zealand White rabbits were anesthetized, instrumented, and studied supine while breathing spontaneously at ambient pressure or during the application of positive or negative airway pressure. Under each of these conditions, the effect of unilateral or bilateral hypoglossal nerve stimulation was investigated. Measurements: Inspiratory flow and tidal volume were measured together with esophageal pressure and the electromyographic activity of diaphragm, alae nasi, and genioglossus muscles. Main results: Anesthesia caused a marked increase in inspiratory resistance, snoring, and in eight rabbits, inspiratory flow limitation. Hypoglossus nerve stimulation was as effective as continuous positive airway pressure in reversing inspiratory flow limitation and snoring. Its effectiveness increased progressively as airway opening pressure was lowered, reached a maximum at $-5 \mathrm{~cm} \mathrm{H}_{2} \mathrm{O}$, but declined markedly at lower pressures. With negative airway opening pressure, airway collapse eventually occurred during inspiration that could be prevented by hypoglossus nerve stimulation. The recruitment characteristics of hypoglossus nerve fibers was steep, and significant upper airway dilating effects already obtained with stimulus intensities 36 to $60 \%$ of maximum. Conclusion: This study supports hypoglossus nerve stimulation as a treatment option for obstructive sleep apnea.
\end{abstract}

Keywords: obstructive sleep apnea; continuous positive airway pressure; continuous negative airway pressure; inspiratory flow limitation; control of breathing

Obstructive sleep apneas and hypopneas (OSAH) are characterized by recurrent episodes of complete or partial obstruction of extrathoracic airways, with reduced ventilation and progressive arterial hypoxemia and hypercapnia, that are usually terminated by brief arousals (1). Although this condition can be effectively treated by means of continuous positive airway pressure (CPAP) applied via a nasal mask (2), this form of treatment is not without side effects and is often poorly tolerated. Strong evidence exists that in most patients with OSAH the obstruction occurs at the level of the oropharynx due to a reduction of tonic and phasic activity of dilating muscles $(3,4)$. Indeed, it has been shown that during sleep, the contraction of the lingual muscles produced by direct hypoglossus nerve stimulation reverses upper airway obstruction in patients with OSAH (5). However, a subsequent

(Received in original form February 7, 2005; accepted in final form May 26, 2005)

Correspondence and requests for reprints should be addressed to François Bellemare, Ph.D., Laboratoire du sommeil, CHUM - Hôtel-Dieu, 3840 St-Urbain, Montréal, PQ, H2W 1T8 Canada. E-mail: bellemaf@colba.net

This article has an online supplement, which is accessible from this issue's table of contents at www.atsjournals.org

Am J Respir Crit Care Med Vol 172. pp 606-612, 2005

Originally Published in Press as DOI: 10.1164/rccm.200502-1900C on June 9, 2005

Internet address: www.atsjournals.org clinical trial (6) revealed a number of problems in a majority of patients, such as electrode breakage, poor synchronization between the stimulator and patients' breathing patterns, and device malfunction, thus showing the need for substantial technical improvements in the therapeutic use of hypoglossus nerve stimulation.

An animal model that mimics the essential features of OSAH could be useful in developing a suitable device for hypoglossus nerve stimulation. The presence of inspiratory flow limitation during tidal breathing would realize a condition very close to OSAH. Studies in rabbits (4) and cats (7-9) have shown that anesthesia depresses the activity of hypoglossus motoneurones markedly more than phrenic nerve activity. Although such an imbalance between the activity of the upper airway dilating muscles and the inspiratory pump muscles could eventually cause tidal inspiratory flow limitation, its occurrence during normal breathing in anesthetized animals has not been investigated. In the present study we show that tidal inspiratory flow limitation can occur in spontaneously breathing, anesthetized rabbits, and that it can be reversed by hypoglossus nerve stimulation. We then addressed the following questions: What is the relation between the strength of hypoglossus nerve stimulation and the relief of inspiratory flow limitation? Is bilateral hypoglossus nerve stimulation more efficient than unilateral stimulation in alleviating upper airway obstruction? How efficient is hypoglossus nerve stimulation both relative to the level of airway obstruction and conventional CPAP?

\section{METHODS}

All surgical and experimental procedures were carried in accordance with the principles and guidelines of the European Community for the use and care of experimental animals. Ten New Zealand White rabbits (body weight: $2.4-3 \mathrm{~kg}$ ) were anesthetized with propofol $\left(5 \mathrm{mg} \cdot \mathrm{kg}^{-1}\right)$ and urethane $\left(1 \mathrm{~g} \cdot \mathrm{kg}^{-1}\right)$, and studied supine. A mouth-occluding nasal mask was held in place with rubber bands and sealed with silicone grease. We measured airflow through the mask with a pneumotachograph (Fleish no. 00; HS Electronics, March-Hugstetten, Germany) and differential pressure transducer (Statham 270; Hewlett Packard, Palo Alto, CA), and airway opening and esophageal pressures with pressure transducers (Model 1290A; Hewlett Packard) connected to a side port of the mask and a balloon-tipped catheter, respectively. The bared 2 to $3 \mathrm{~mm}$ of four pairs of fine, Teflon-coated wires were inserted into the diaphragm, right alae nasi, right and left genioglossus muscles, and connected to a custom-built apparatus that amplified, rectified, and integrated (time constant $=0.1 \mathrm{~s}$ ) the electromyographic $(\mathrm{EMG})$ activity. All signals were sampled at $200 \mathrm{~Hz}$ by a 12-bit A/D converter (AT MIO16E-10; National Instruments, Austin, TX) and stored on computer. Following a ventral approach, the hypoglossus nerves were isolated, placed on small, half-cuff platinum bipolar electrodes (10) connected to a stimulator (S44F; Grass Instrument, Quincy, MA), and covered with warm mineral oil. Square wave stimuli, $0.1 \mathrm{~ms}$ in duration and 0.3 to $13 \dot{\mathrm{V}}$ in amplitude, were delivered as single pulses or at $70 \mathrm{~Hz}$.

\section{Protocol}

For each nerve, the relationship between stimulus strength and amplitude of the compound motor action potential of the genioglossus muscle was determined first. Thereafter, records were taken during periods of spontaneous breathing at ambient pressure (10 rabbits) and at various 
levels of CPAP and continuous negative airway pressure (CNAP) adjusted by means of a flow-through system connected to the pneumotachograph (eight rabbits). At each level of airway pressure, maximal stimulation at $70 \mathrm{~Hz}$ was applied three times for three consecutive breaths, while unilateral stimulation of various strengths up to maximum were performed only during ambient pressure breathing. Before each test, the airways were thoroughly suctioned. Additional details can be found in online supplement.

\section{Data Analysis}

For each condition, nine control breaths and nine stimulated breaths were analyzed: mean inspiratory flow, tidal volume by numerical integration of the flow signal, tidal esophageal pressure swing, peak diaphragm, alae nasi and genioglossal EMG activity, and inspiratory duration from onset to peak diaphragm EMG activity. Dynamic pressure during inspiration, that is, the transpulmonary pressure related to inspiratory pulmonary resistance, was computed from the inspiratory limb of the ensemble-averaged esophageal pressure-lung volume loops according to the subtraction method (11).

Values are presented as mean $\pm \mathrm{SE}$. Comparisons between conditions were made using repeated-measures ANOVA and post hoc Bonferroni test with the hypoglossus nerve stimulation factor nested within the applied pressure factor when appropriate (12). A univariate $t$ test was employed to evaluate the effects of different levels of stimulation on outcome variables. Linear regression was computed with the leastsquare method. The level of significance was taken at $p<0.05$.

\section{RESULTS}

Under all conditions, the onset of EMG activity was essentially simultaneous in all muscles, whereas peak alae nasi and genioglossus EMG activity preceded that of the diaphragm (Figure 1). Under control condition at ambient pressure, peak alae nasi and genioglossus EMG activity occurred $90 \pm 25$ and $97 \pm 19 \mathrm{~ms}$ before peak diaphragm EMG activity, respectively $(p<0.002)$. Although diaphragm and alae nasi EMG activity was present in all animals and conditions, in one rabbit genioglossus EMG activity was always absent and in an other animal it appeared only during CNAP breathing.

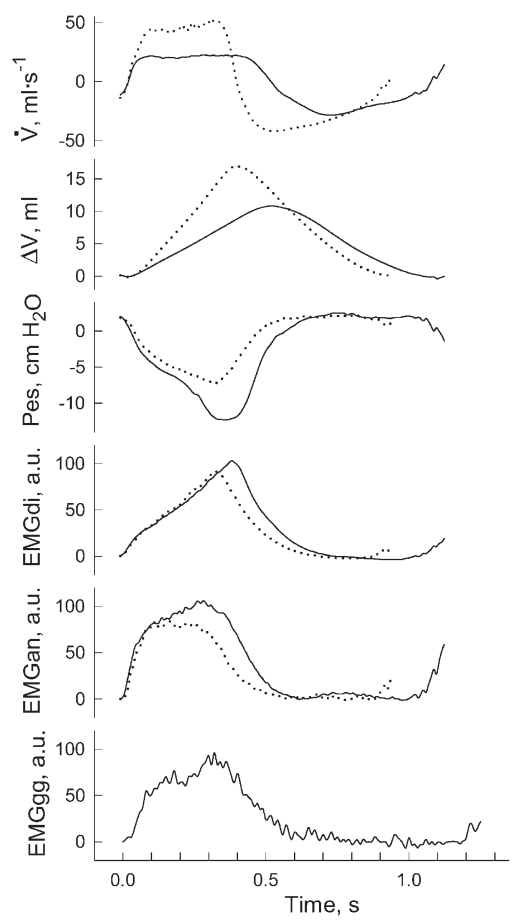
aligned at the onset of inspiratory flow.

Fure 1. Ensemble-averlung volume changes $(\Delta)$, esophageal pressure alae nasi (an), and genioglossus (gg) integrated ity (EMG) in an anesthetized rabbit breathing at ambient pressure with (ine) and without bilateral hypoglossus nerve stimulation. All traces were (continuous line) maximal,
During control breathing at ambient pressure, the relationships between inspiratory flow and dynamic transpulmonary pressure were markedly curvilinear in all rabbits, thus showing a progressive increase in pulmonary resistance throughout inspiration (Figure 2). In eight rabbits, the slope of this relationship became eventually nil or even negative, indicating the occurrence of inspiratory flow limitation. The values of dynamic transpulmonary pressure at which inspiratory flow became limited ranged from -3.0 to $-10.3 \mathrm{~cm} \mathrm{H}_{2} \mathrm{O}$, with a mean value of $-6.1 \pm 0.9 \mathrm{~cm}$ $\mathrm{H}_{2} \mathrm{O}$. All rabbits were snoring, as shown by the presence of highfrequency oscillations in the mask pressure trace during inspiration (Figure 3).

\section{Stimulations during Ambient Pressure Breathing}

Maximal stimulations. In all rabbits, the relationship between inspiratory flow and dynamic transpulmonary pressure became essentially linear or moderately curved with maximal, bilateral hypoglossus nerve stimulation, indicating a marked reduction in pulmonary inspiratory resistance (Figure 2). Inspiratory flow limitation was abolished (Figure 2) and snoring also disappeared (Figure 3). Inspiratory flow and tidal volume increased, whereas esophageal pressure swings, diaphragm and alae nasi peak EMG activity, and inspiratory duration decreased (Figure 1), all these changes being significant (Table 1). Hypoglossus nerve stimulation had no effect on the rate of rise of integrated diaphragm and alae nasi EMG activity, the fall in peak EMG activity being therefore due to shortening of inspiratory duration caused by the increase in tidal volume (Figure 1 and Table 1).

Maximal stimulation of the left or right hypoglossus nerve caused similar changes of all variables (Table 1). These changes were not significantly different from those obtained with maximal, bilateral hypoglossus nerve stimulation. Whereas no difference in genioglossus EMG activity occurred on the stimulated side between unilateral and bilateral stimulation, that of the contralateral side increased systematically with unilateral stimulation, averaging $20 \%$ of maximum.

Submaximal unilateral stimulations. The recruitment threshold of the 20 hypoglossus nerves was $3.2 \pm 0.4 \mathrm{~V}$ (range, 0.3-8 V). Maximum recruitment occurred at $6.4 \pm 0.8 \mathrm{~V}$ (range, 1.8-13 V). All recruitment curves showed a steep slope above threshold,

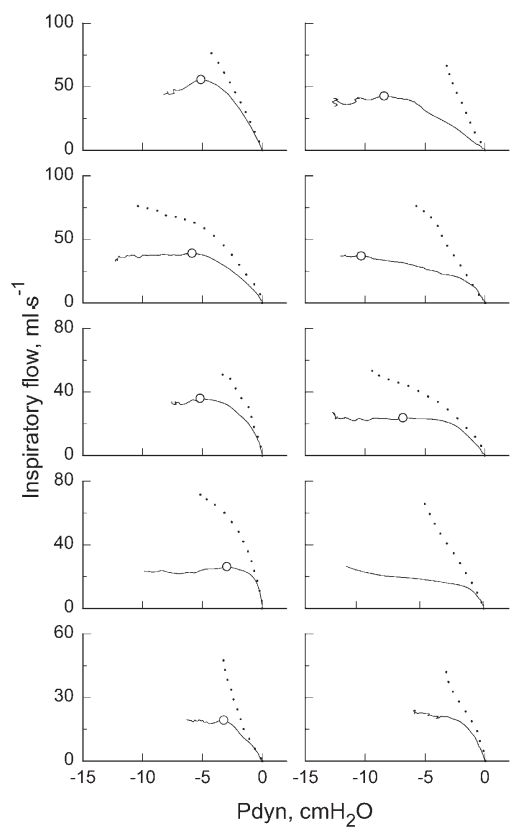

Figure 2. The relationship between inspiratory airflow and dynamic transpulmonary pressure (Pdyn) in anesthetized rabbits breathing at ambient pressure with (dotted line) and without (continuous line) maximal, bilateral hypoglossus nerve stimulation. Circles indicate onset of inspiratory flow limitation. 

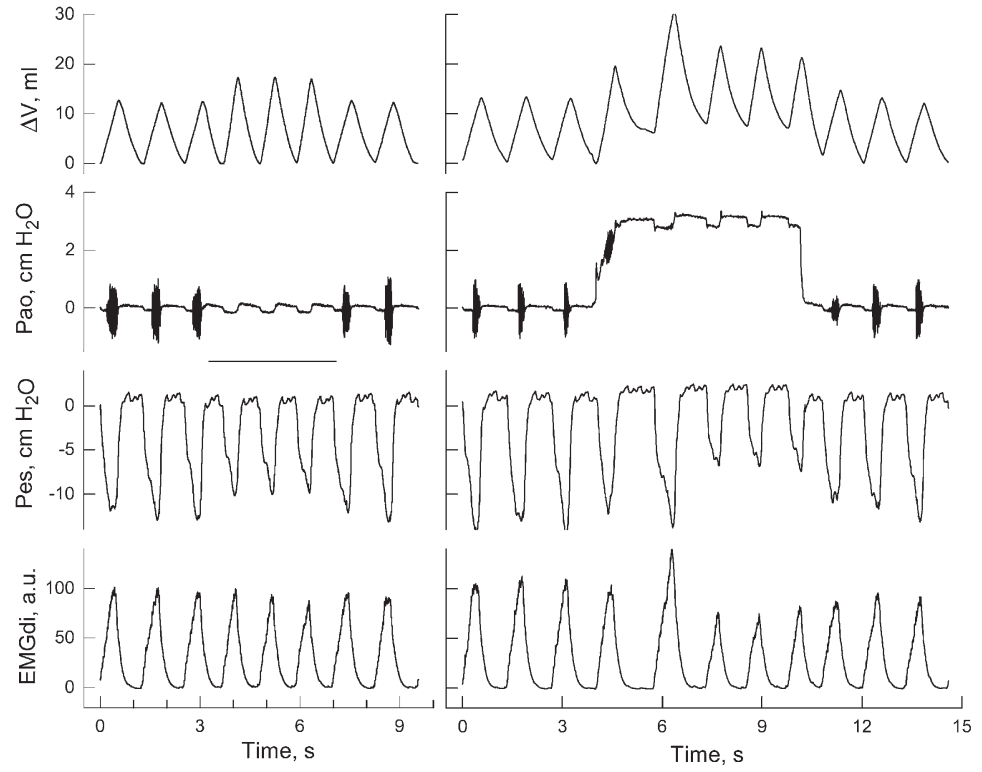

Figure 3. Records of lung volume changes $(\Delta \mathrm{V})$, mask (Pao) and esophageal pressure (Pes), and diaphragm integrated electromyographic activity (EMGdi) in an anesthetized rabbit breathing at ambient pressure with (marked by horizontal bar under Pao tracing) and without maximal, bilateral hypoglossus nerve stimulation (left) or with and without continuous positive applied pressure (right). Snoring is indicated by the high frequency oscillations of mask pressure. and for 19 nerves, complete recruitment occurred at a voltage less than twice the threshold (Figure 4).

Although for 13 hypoglossus nerves, unilateral stimulation produced the same qualitative effects as bilateral stimulation at all levels of stimulus strength, for 7 nerves constricting effects occurred at some levels of submaximal stimulation. In spite of this, a clear gradation in the response to unilateral stimulation was observed when all data were grouped into four ranges of genioglossus EMG activity, namely 0 to $35 \%, 36$ to $60 \%, 61$ to $85 \%$, and 86 to $100 \%$ of maximal activity. As shown in Figure 5, the changes in inspiratory flow, esophageal pressure, and tidal volume became significant when genioglossus EMG activity was between 36 and $60 \%$ maximum, those in diaphragm and alae nasi peak EMG activity when genioglossus EMG activity was between 61 and $85 \%$ of maximum $(\mathrm{p}<0.05)$.

\section{CPAP and CNAP Breathing}

With increasing CPAP (Figure 6), inspiratory flow and tidal volume increased, whereas esophageal pressure swings, diaphragm, alae nasi, and genioglossal peak EMG activity decreased ( $p<0.01)$, the fall of peak EMG activity being due to shortening of inspiratory duration. The mechanical variables and diaphragm EMG activity tended to plateau at CPAP greater than or equal to $3 \mathrm{~cm} \mathrm{H}_{2} \mathrm{O}$ when inspiratory flow limitation (Figure 7) and snoring (Figure 3 ) were absent in all animals, and became similar to the corresponding values with hypoglossus nerve stimulation at ambient pressure. Alae nasi and genioglossus peak EMG activity showed, however, a small, further decrease with CPAP greater than $3 \mathrm{~cm} \mathrm{H}_{2} \mathrm{O}$.

With increasing CNAP (Figure 6), inspiratory flow and tidal volume decreased, whereas esophageal pressure swings, diaphragm, alae nasi, and genioglossus peak EMG activity increased ( $\mathrm{p}<0.001$ ), the latter being due both to an increased rate of rise of EMG activity and to the lengthening of inspiratory duration. The increased rate of rise of diaphragm EMG activity reflected the hypoventilation, and hence the increased chemical drive, caused by CNAP. However, because CNAP was applied for short periods, the mean rate of rise of diaphragm EMG activity with the lowest CNAP was, on average, only 1.15 times that during ambient pressure breathing. The increase in alae nasi and genioglossus peak EMG activity did not prevent inspiratory flow limitation, which with CNAP of less than or equal to $-2 \mathrm{~cm} \mathrm{H}_{2} \mathrm{O}$ occurred also in the two rabbits that were non-flow limited during breathing at ambient pressure (Figure 2). In spite of increasing alae nasi and genioglossus EMG activity with increasing CNAP (Figure 6), inspiratory flow limitation occurred at progressively lower flows, and with CNAP less than or equal to $5 \mathrm{~cm} \mathrm{H}_{2} \mathrm{O}$, closure of extrathoracic airways occurred in all rabbits during at least part of inspiration (Figure 7).

TABLE 1. EFFECTS OF MAXIMAL BILATERAL AND UNILATERAL HYPOGLOSSUS NERVE STIMULATION IN TEN ANESTHETIZED RABBITS BREATHING AT AMBIENT PRESSURE

\begin{tabular}{|c|c|c|c|c|}
\hline & Control & Bilateral & Right & Left \\
\hline$\dot{\mathrm{V}}_{\mathrm{l}}, \mathrm{ml} \cdot \mathrm{s}^{-1}$ & $28.1 \pm 2.8$ & $50.0 \pm 3.6^{*}$ & $45.7 \pm 3.5^{*}$ & $45.8 \pm 3.7^{*}$ \\
\hline$\Delta$ Pes, $\mathrm{cm} \mathrm{H}_{2} \mathrm{O}$ & $-14.7 \pm 1.4$ & $-9.6 \pm 0.7^{*}$ & $-9.6 \pm 1.1^{*}$ & $-10.7 \pm 1.0^{*}$ \\
\hline $\mathrm{V}_{\mathrm{T}}, \mathrm{ml}$ & $13.1 \pm 0.8$ & $18.3 \pm 0.7^{*}$ & $18.5 \pm 0.9^{*}$ & $17.6 \pm 1.0^{*}$ \\
\hline peak EMGdi, \% & 100 & $80.3 \pm 7.7^{\star}$ & $88.3 \pm 2.2^{*}$ & $86.7 \pm 1.5^{*}$ \\
\hline peak EMGan, \% & 100 & $68.7 \pm 6.8^{*}$ & $78.8 \pm 1.9^{*}$ & $88.2 \pm 3.8^{*}$ \\
\hline $\mathrm{TI}, \mathrm{s}$ & $0.43 \pm 0.03$ & $0.37 \pm 0.03^{*}$ & $0.36 \pm 0.2^{*}$ & $0.37 \pm 0.03^{*}$ \\
\hline
\end{tabular}

Definition of abbreviations: $\Delta$ Pes = tidal esophageal pressure swing; EMGdi and EMGan = diaphragm and alae nasi peak integrated electromyographic activity, expressed as percent of control; $\mathrm{T}_{\mathrm{I}}=$ inspiratory duration; $\dot{\mathrm{V}}_{\mathrm{I}}=$ mean inspiratory flow; $\mathrm{V}_{\mathrm{T}}=$ tidal volume. Values are mean $\pm \mathrm{SE}$.

${ }^{*} \mathrm{p}<0.05$ vs. control. 


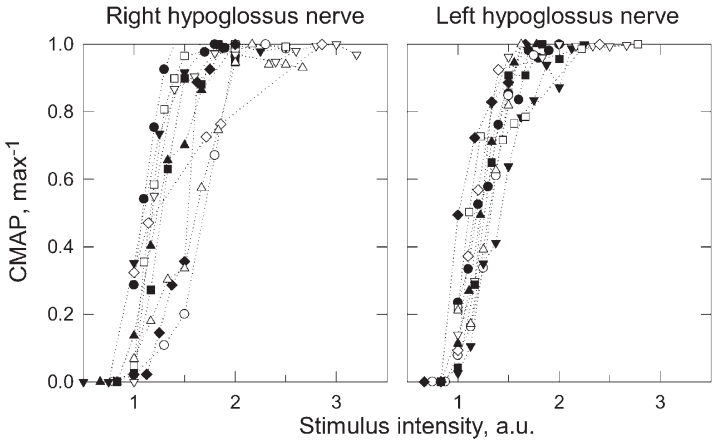

Figure 4. The relationship between stimulus intensity, expressed as a fraction of recruitment threshold, and amplitude of the compound motor action potential (CMAP), expressed as percent of maximum, during unilateral hypoglossus nerve stimulation. Different symbols indicate different rabbits.

\section{Stimulations during CPAP and CNAP Breathing}

The effects of hypoglossus nerve stimulation during CPAP breathing, though qualitatively similar to those at ambient pressure, became trivial at CPAP greater than or equal to $3 \mathrm{~cm} \mathrm{H}_{2} \mathrm{O}$ (Figure 6). At these levels of CPAP, no significant differences were observed in the inspiratory flow-dynamic transpulmonary pressure relationship of breaths with and without hypoglossus nerve stimulation (Figure 7). For all variables, no significant difference occurred during hypoglossus nerve stimulation among all levels of CPAP, or between CPAP and ambient pressure breathing. By contrast, at all levels of CNAP hypoglossus nerve stimulation increased inspiratory flow and tidal volume, and decreased esophageal pressure swings, and diaphragm and alae nasi peak EMG activity (Figure 6), all these changes being significant $(\mathrm{p}<0.006)$. Hypoglossus nerve stimulation abolished inspiratory flow limitation in all animals except at the lowest CNAP, when it was, however, capable to prevent extra-thoracic airway closure (Figure 7).

\section{Effectiveness of Hypoglossus Nerve Stimulation}

The effectiveness of maximal, bilateral hypoglossus nerve stimulation evaluated from mean inspiratory flow and tidal volume
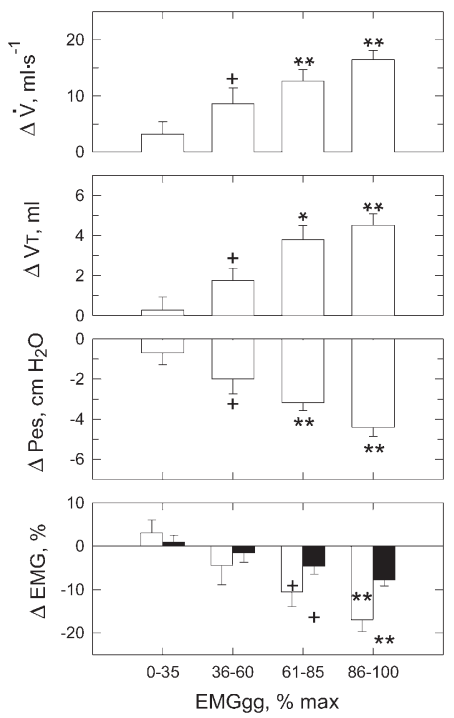

Figure 5. The changes in mean inspiratory flow $\left(\dot{V}_{I}\right)$, tidal volume $(\mathrm{V} T)$, esophageal pressure swings (Pes), and diaphragm (filled bars) and alae nasi (open bars) peak integrated electromyographic activity (EMG) due to unilateral hypoglossus nerve stimulation of different intensities delivered during ambient pressure breathing in 10 rabbits. Data obtained with left and right hypoglossus nerve stimulation were pooled. Symbols indicate significant changes $\left({ }^{+} p<0.05\right.$; $\left.{ }^{*} p<0.01 ;{ }^{* *} p<0.001\right)$. For definition of the levels of stimulus intensity see text.

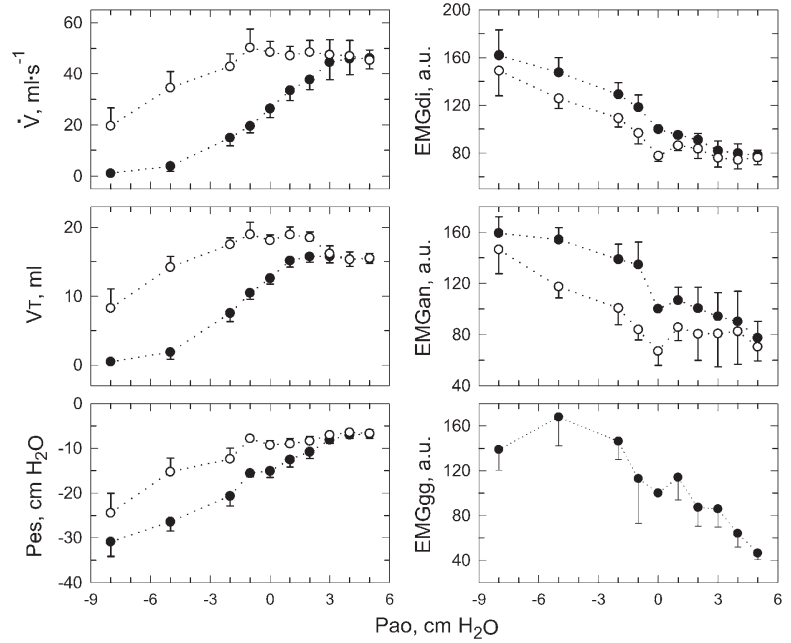

Figure 6. Mean inspiratory flow $\left(\dot{V}_{1}\right)$, tidal volume $\left(V_{T}\right)$, esophageal pressure swings (Pes), and peak diaphragm (di), alae nasi (an) and genioglossus (gg) electromyographic activity (EMG) in eight rabbits breathing at various airway opening pressures (Pao) with (open circles) and without maximal, bilateral hypoglossus nerve stimulation (filled circles). Bars are SE.

changes, depended on airway opening pressure. As shown by the vertical distance between open and closed symbols in Figure 6 , these changes decreased with increasing CPAP, becoming small and not significant at $\mathrm{CPAP}>3 \mathrm{~cm} \mathrm{H}_{2} \mathrm{O}$, whereas they progressively increased with decreasing airway opening pressure to $-5 \mathrm{~cm} \mathrm{H}_{2} \mathrm{O}$, and then markedly declined, being still significant at CNAP of $-8 \mathrm{~cm} \mathrm{H}_{2} \mathrm{O}(\mathrm{p}<0.05)$.
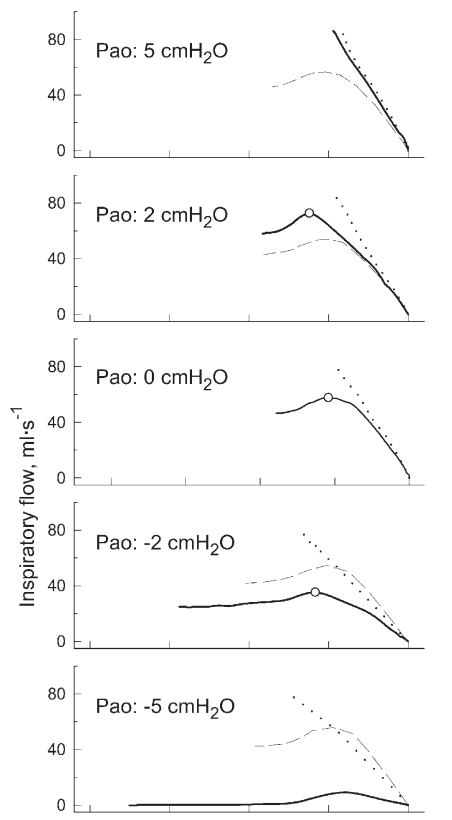

Figure 7. The relationship between inspiratory airflow and dynamic transpulmonary pressure (Pdyn) in a representative, anesthetized rabbit breathing at various airway opening pressures (Pao) with (dotted line) and without maximal, bilateral hypoglossus nerve stimulation (continuous line). The dashed lines indicate the relationship with Pao $=0 \mathrm{~cm} \mathrm{H} \mathrm{H}_{2} \mathrm{O}$ that occurred just before continuous positive or negative pressure breathing. Circles indicate onset of inspiratory flow limitation.

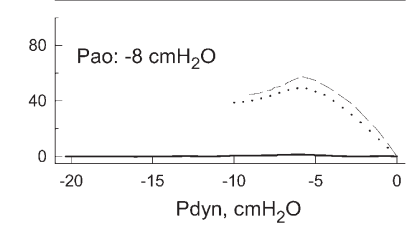


Vagal mechanisms controlling inspiratory duration were also affecting the effectiveness of hypoglossus nerve stimulation. Indeed, the fall of effective pulmonary resistance caused by hypoglossus nerve stimulation, allowed a more rapid attainment of the volume-dependent vagal threshold for termination of inspiration, thus shortening the inspiratory duration. Because the rate of rise of diaphragm EMG activity, as well as of alae nasi and genioglossus EMG activity, were unchanged (Figure 1), the shortening of inspiratory duration decreased both the peak EMG activity of these muscles and tidal volume. As a consequence, the relationship between peak EMG activity of diaphragm, alae nasi, and genioglossus muscles was described by a linear function with a slope not significantly different from unity, independent of hypoglossus nerve stimulation and airway opening pressure (Figure 8).

\section{DISCUSSION}

The major findings are (1) anesthetized, spontaneously breathing supine rabbits exhibit very high inspiratory flow resistance and snoring, inspiratory flow limitation occurring in most of them; (2) hypoglossus nerve stimulation and conventional CPAP are equally effective in eliminating inspiratory flow limitation and snoring; (3) unilateral hypoglossus nerve stimulation is as effective as bilateral stimulation; (4) significant upper airway dilating effects can be obtained at stimulus intensities 36 to $60 \%$ of maximum; and (5) the effectiveness of hypoglossus nerve stimulation, as evaluated from the induced mean inspiratory flow and tidal volume changes, depends on airway pressure, maximal response occurring at $-5 \mathrm{~cm} \mathrm{H}_{2} \mathrm{O}$.

\section{Critique of the Model}

The present animal model differs from human OSAH as there are no sleep stage transitions, no apneic episodes, and no arousals are possible. Nevertheless, the pattern of inspiratory flow limitation occurring in most rabbits resembles that seen in patients with OSAH and in snorers (13). Furthermore, obstructive apneas were produced by lowering airway pressure, allowing the effectiveness of hypoglossus nerve stimulation to be assessed under conditions of complete airway collapse. The mechanisms of inspiratory flow limitation in the present model should be the same as in many patients with OSAH, namely an uncoupling between activation of upper airway dilating and inspiratory pump muscles, with the tongue relapsing against the walls of the pharynx as the intraluminal pressure drops during inspiration $(3,4)$. Indeed, inspiratory flow limitation and snoring were abolished by hypoglossus nerve stimulation in all rabbits. Inspiratory flow limitation was not due to abnormally increased upper airway collapsibility, as the dynamic transpulmonary pressure at which inspiratory flow limitation occurred $\left(-6.1 \pm 0.9 \mathrm{~cm} \mathrm{H}_{2} \mathrm{O}\right)$ is close to the average critical closing pressure $\left(-5.3 \pm 1 \mathrm{~cm} \mathrm{H}_{2} \mathrm{O}\right)$ found

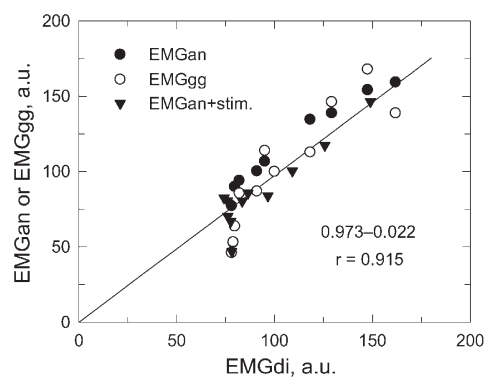

Figure 8. The relationship between diaphragm (di) peak integrated electromyographic activity (EMG) and alae nasi (an) and genioglossus (gg) peak integrated EMG activity during breathing at ambient pressure (control) and at various levels of positive (CPAP) and negative airway opening pressure (CNAP) with or without maximal bilateral hypoglossal nerve stimulation (see key to symbols). Slope ( \pm SE) and correlation coefficient are indicated. in the isolated upper airway preparation of decerebrate cats (14). Because the present model mimics the most important features of human OSAH, it should prove useful in the development and testing of permanent hypoglossus nerve implants, besides allowing the study of the effects of hypoglossus nerve stimulation on upper airway mechanics and motor control during sleep.

Experiments were performed in the supine position because of easier access to the hypoglossus nerves. The mechanical properties and neural control of upper airways could differ in other body positions, because of the effect of gravity. However, rabbits that exhibited tidal flow limitation and snoring in the supine posture did so also in the lateral decubitus, and in the rabbits in which this was tested, hypoglossus nerve stimulation prevented inspiratory flow limitation in both postures.

Anesthesia was induced with propofol and maintained with urethane, a combination chosen because of its stable and longlasting effects. However, the present findings are unlikely to depend on the type of anesthesia, because a depressant effect on hypoglossus nerve activity has been observed with several other anesthetics (4, 7-9). The EMG activity of genioglossus muscle was absent in two rabbits under control condition, whereas substantial activity of alae nasi was always present in all animals; this could suggest that among the motoneurones innervating the upper airway dilating muscles, those of the hypoglossus nucleus are more prone to the depressant effects of anesthesia.

Maximal whole nerve stimulation produced dilating effects in all rabbits. On the other hand, hypoglossus nerve stimulation also activates the retractor muscles of the tongue, which cause upper airway obstruction when selectively stimulated $(15,16)$, and hence antagonize the action of the protruder muscles. Indeed, a constricting effect was occasionally observed in the present animals at some levels of submaximal unilateral stimulation, probably because in those cases the nerve fibers supplying the retractor muscles were closer to the stimulating electrodes, and hence more easily recruited than those supplying the protruder muscles. Thus, with submaximal unilateral hypoglossus nerve stimulation, appropriate electrode positioning becomes an important issue. Interestingly, in previous studies in cats $(17,18)$ and humans (5), no constricting response has been found with whole nerve stimulation at stimulus strengths just above threshold. It should be noted, however, that in those studies the threshold was assessed by visual appreciation of tongue motion rather than genioglossus EMG activity. Given the steepness of the recruitment curves (Figure 4) and the co-activation of antagonist muscles, the mechanical threshold is likely to exceed the EMG threshold, thus explaining why a constricting response was not observed previously with low intensity stimulation. Although the occurrence of constricting effects with submaximal stimulation should not be overlooked, a significant dilating response is usually obtained when genioglossus activation is greater than $35 \%$ maximum (Figure 5).

\section{The Effects of Hypoglossus Nerve Stimulation}

The effects of hypoglossus nerve stimulation in the present rabbits are qualitatively similar to those observed in patients with severe OSAH during sleep. In the five patients studied by Eisele and coworkers (5), inspiratory flow increased by $185 \mathrm{ml} \cdot \mathrm{s}^{-1}$ or about $50 \%$ during unilateral stimulation, whereas in rabbits it increased by $22 \mathrm{ml} \cdot \mathrm{s}^{-1}$ or $78 \%$ during bilateral stimulation and by $18 \mathrm{ml} \cdot \mathrm{s}^{-1}$ or $64 \%$ during unilateral stimulation. The small difference between inspiratory flow changes caused by unilateral and bilateral stimulation indicates that the effects of unilateral stimulation are not additive, possibly because relatively little force is required to displace the tongue and normalize upper 
airway resistance. Moreover, in all animals, a significant EMG activity ( $\sim 20 \%$ of maximum) was recorded on the contralateral side during unilateral maximal stimulation. It is unlikely that this activity could reflect a contamination form the active side, as it remained essentially unchanged in spite of electrodes repositioning. It more likely reflects the presence of cross-innervation, as demonstrated in the dog (19). This further explains why the responses to unilateral stimulation are not additive.

With increasing CPAP, flow limitation occurred at higher levels of inspiratory flow and was eventually abolished at CPAP greater than or equal to $3 \mathrm{~cm} \mathrm{H}_{2} \mathrm{O}$ (Figure 7), whereby both mean inspiratory flow and tidal volume increased relative to ambient pressure breathing (Figure 6). In contrast, with CNAP flow limitation occurred at progressively lower levels of inspiratory flow and airway collapse eventually took place during inspiration (Figure 7), whereby both mean inspiratory flow and tidal volume decreased, in spite of the concomitant, substantial increase in upper airway muscle activation (Figure 6). On the other hand, the changes in mean inspiratory flow and tidal volume due to hypoglossus nerve stimulation increased in a roughly linear fashion as airway opening pressure was lowered below $3 \mathrm{~cm}$ $\mathrm{H}_{2} \mathrm{O}$ (Figure 6), in line with the observation that in cats the changes in the cross-section area of the rostral oropharynx caused by hypoglossus nerve stimulation increase as the intraluminal pressure is lowered from 6 to $-6 \mathrm{~cm} \mathrm{H}_{2} \mathrm{O}(18)$, probably reflecting the force-length relationship of the genioglossus muscle (20). With airway opening pressure greater than or equal to $-5 \mathrm{~cm} \mathrm{H}_{2} \mathrm{O}$ and maximal hypoglossus nerve stimulation, inspiratory flow limitation was absent (Figure 7), the inspiratory flow being, therefore, set by the available dynamic transpulmonary pressure and pulmonary resistance. Both the dynamic transpulmonary pressure, due to enhanced chemical drive and inspiratory pump muscle activation, and pulmonary resistance, due to lowered transmural pressure, increased with decreasing airway opening pressure (Figure 7). Because the latter increased relatively more than the former, mean inspiratory flow and tidal volume decreased with decreasing airway opening pressure during hypoglossus nerve stimulation, but much less than during nonstimulated, flow-limited inspirations. This explains the increasing effectiveness of hypoglossus nerve stimulation as airway opening pressure was lowered from 3 to $-5 \mathrm{~cm} \mathrm{H}_{2} \mathrm{O}$ (Figure 6). With the lowest CNAP, however, maximal hypoglossus nerve stimulation could not prevent inspiratory flow limitation, and its effectiveness in increasing mean inspiratory flow and tidal volume was therefore substantially reduced.

Nasal CPAP is the conventional treatment of OSAH to which hypoglossus nerve stimulation should be compared. This comparison has never been performed in clinical trials $(5,6)$. In the rabbits used in the present study, hypoglossus nerve stimulation at ambient airway pressure was as effective as CPAP in decreasing inspiratory pulmonary resistance and eliminating snoring and inspiratory flow limitation. Indeed, the same mean inspiratory flow and tidal volume were achieved with hypoglossus nerve stimulation at ambient airway pressure and CPAP of 2 to $3 \mathrm{~cm}$ $\mathrm{H}_{2} \mathrm{O}$, when these parameters attained their largest values (Figure 6 ). Actually, the increase in tidal volume produced by hypoglossus nerve stimulation and CPAP could have been even larger if it had not caused a concomitant decrease of inspiratory duration, and hence of peak diaphragm EMG activity (Figure 1). These effects were expected on the basis of the lung volume related, phasic and tonic vagal activity controlling inspiratory duration (21) and the observed changes in end-inspiratory lung volume produced by hypoglossus nerve stimulation or CPAP at constant chemical drive. Apparently, the same mechanisms are operating in snorers during sleep, in whom resistive unloading by means of CPAP (22) or administration of a low density gas mixture (23) has been shown to increase tidal volume and decrease peak diaphragm EMG activity and inspiratory duration.

Maximal hypoglossus nerve stimulation as employed in the present study may cause arousal in sleeping animals and humans, and may not be tolerated. In human studies $(5,6)$, stimulus strength was adjusted to maximize inspiratory flow without causing arousal, but the recruitment characteristics of hypoglossus nerve fibers were not investigated. In one study in which the response to varying stimulus intensity was evaluated on the basis of mechanical effects (5), the arousal threshold (1.9 V) was 1.7 times the recruitment threshold $(1.1 \mathrm{~V})$. In rabbits, an essentially maximal mechanical response was obtained with stimulus strengths that caused an average genioglossus peak EMG activity $73 \%$ of maximal (Figure 5). According to the stimulus-response curves of Figure 4, such stimulus strengths correspond to $2 \cdot 0.73=1.5$ times the recruitment threshold voltage, maximal activation of genioglossus muscle being always reached with stimulus intensity twice the recruitment threshold voltage. Hence, if the recruitment characteristics of hypoglossus nerve fibers in man were similar to those depicted in Figure 4, maximal mechanical effects of hypoglossus nerve stimulation should be obtainable in man without causing arousal. In the patients of Schwartz and colleagues (6) obstructive events persisted with hypoglossus nerve stimulation, suggesting that stimulation was less efficient in their patients than in the present rabbits, possibly because of differences in stimulus intensity, electrode placement, or severity of airflow obstruction.

The present findings may have important implications for the use of hypoglossus nerve stimulation as a treatment of OSAH, as well as for the design of nerve implants. Indeed, because an essentially full dilating effect was already obtained with unilateral hypoglossus nerve stimulation, little additional benefits should be expected with bilateral stimulation. Moreover, no laterality was found in the present study, the effects of left and right hypoglossus nerve stimulation being essentially the same (Table 1). Finally, recruitment of other upper airway muscles should also cause no additional benefits, because no dilating effects beyond those with maximal bilateral hypoglossus nerve stimulation were produced by CPAP (Figures 6 and 7). A unilateral implant is certainly a simpler device than a bilateral implant, and requires less extensive surgery with lower risks. On the other hand, a bilateral implant has the advantage of allowing alternation between left and right hypoglossus nerve stimulation, thus reducing the risks of nerve or muscle damage or fatigue. Furthermore, although no additive effects occurred with maximal stimulation, it is possible that such effects could take place with submaximal stimulation or at lower intraluminal pressures, when a greater force is required to displace the tongue. Clearly, all these issues need to be investigated before a recommendation can be made as to the most suitable approach for hypoglossus nerve stimulation.

In conclusion, this study has shown that tidal inspiratory flow limitation and snoring occur in anesthetized rabbits breathing spontaneously at ambient pressure, thus resembling the condition encountered in patients with OSAH. Both inspiratory flow limitation and snoring were abolished by hypoglossus nerve stimulation, the effectiveness of which is essentially the same during unilateral and bilateral maximal stimulation and similar to or even greater than that of CPAP. With CNAP, hypoglossus nerve stimulation was still capable to substantially increase inspiratory flow and tidal volume, abolishing inspiratory flow limitation in most circumstances, and preventing the collapse of extrathoracic airways. The recruitment characteristics of hypoglossus nerve fibers was very steep, thus favoring the implementation of hypoglossus nerve stimulation as a therapeutic option for OSAH. The present animal model should, therefore, prove useful in the 
development of suitable nerve implants and in the investigation of still open questions.

Conflict of Interest Statement: None of the authors have a financial relationship with a commercial entity that has an interest in the subject of this manuscript.

Acknowledgment: The present study was performed during a sabbatical leave of F.B. to Istituto di Fisiologia Umana I, Università degli Studi di Milano. The support of Ministero dell'Istruzione, dell'Università e della Ricerca Scientifica of Italy is gratefully acknowledged.

\section{References}

1. American Academy of Sleep Medicine Task Force. Sleep-related breathing disorders in adults: Recommendations for syndrome definition and measurement techniques in clinical research. Sleep 1999;22:667-688.

2. Sullivan CE, Issa FG, Berthon-Jones M, Eves L. Reversal of obstructive sleep apnoea by continuous positive airway pressure applied through the nares. Lancet 1981;1:862-865.

3. Remmers JE, Degroot WJ, Sauerland EK, Anch AM. Pathogenesis of upper airway occlusion during sleep. J Appl Physiol 1978;44:931-938.

4. Brouillette RT. A neuromuscular mechanism maintaining extrathoracic airway patency. J Appl Physiol 1979;46:772-779.

5. Eisele DW, Smith PL, Alam DS, Schwartz AR. Direct hypoglossal nerve stimulation in obstructive sleep apnea. Arch Otolaryngol Head Neck Surg 1997;123:57-61.

6. Schwartz AR, Bennett ML, Smith PL, De Backer W, Hedner J, Boudewijns A, Van de Heyning P, Ejnell H, Hochban W, Knaack L, et al. Therapeutic electrical stimulation of the hypoglossal nerve in obstructive sleep apnea. Arch Otolaryngol Head Neck Surg 2001;127:1216-1223.

7. Nishino T, Shirahata M, Yonezawa T, Honda Y. Comparison of changes in the hypoglossal and the phrenic nerve activity in response to increasing depth of anesthesia in cats. Anesthesiology 1984;60:19-24.

8. Hwang JC, St John WM, Bartlett D Jr. Respiratory-related hypoglossa nerve activity: influence of anesthetics. J Appl Physiol 1983;55:785-792.

9. Bennett FM, St John W. Anesthesia selectively reduces hypoglossal nerve activity by actions upon the brain stem. Pflugers Arch 1984;401:421423.

10. Boyer S, Sawan M, Abdel-Gawad M, Robin S, Elhilali MM. Implantable selective stimulator to improve bladder voiding: design and chronic experiments in dogs. IEEE Trans Rehabil Eng 2000;8:464-470.

11. Von Neergard K, Wirz K. Die Messung der Strömungswiderstände in den Atemwegen des Menschen, insbesondere bei Asthma und Emphysem. Z Klin Med 1927;195:51-82.

12. Sokal R, Rohlf F. Biometry, 2nd ed. New York: W. H. Freeman and Co; 1981.

13. Clark S, Wilson C, Satoh M, Pegelow D, Dempsey JA. Assessment of inspiratory flow limitation invasively and non-invasively during sleep. Am J Respir Crit Care Med 1998;158:713-722.

14. Schwartz AR, Thut DC, Russ B, Seelagy M, Yuan X, Brower RG, Permutt S, Wise RA, Smith PL. Effect of electrical stimulation of the hypoglossal nerve on airflow mechanics in the isolated upper airway. Am Rev Respir Dis 1993;147:1144-1150.

15. Schwartz AR, Eisele DW, Hari A, Testerman R, Erickson DJ, Smith PL. Electrical stimulation of the lingual musculature in obstructive sleep apnea. J Appl Physiol 1996;81:643-652.

16. Fuller DD, Williams JS, Janssen PL, Fregosi RF. Effect of co-activation of tongue protrudor and retractor muscles on tongue movements and pharyngeal airflow mechanics in the rat. J Physiol 1999;519:601-613.

17. Kuna ST. Effects of pharyngeal muscle activation on airway size and configuration. Am J Respir Crit Care Med 2001;164:1236-1241.

18. Kuna ST, Brennick MJ. Effects pf pharyngeal muscle activation on airway pressure-area relationships. Am J Respir Crit Care Med 2002;166:972977.

19. Chibuzo GA, Cummings JF. An enzyme tracer study of the organization of the somatic motor center for the innervation of different muscles of the tongue: evidence for two sources. J Comp Neurol 1982;205:273-281.

20. Brennick MJ, Parisi RA, England SJ. Influence of preload and afterload on genioglossus muscle length in awake goats. Am J Respir Crit Care Med 1997;155:2010-2017.

21. Bradley GW, von Euler C, Martille I, Roos B. A model of the central and reflex inhibition of inspiration in the cat. Biol Cybern 1975;19:105-116

22. Henke KG, Dempsey JA, Kowitz JM, Skatrud J. Effects of sleep-induced increases in upper airway resistance on ventilation. $J$ Appl Physiol 1990;69:617-624.

23. Skatrud J, Dempsey JA, Badr S, Begle RL. Effect of airway impedance on $\mathrm{CO} 2$ retention and respiratory muscle activity during NREM sleep. J Appl Physiol 1988;65:1676-1685. 